\title{
Digital ImAge CoRrelation ANALYSis OF LAMINATED BAMBoO UNDER TRANSVERSE COMPRESSION
}

\author{
Ben Tinkler-Davies ${ }^{a}$, Darshil U. Shah ${ }^{b *}$ \\ ${ }^{a}$ Division of Mechanics, Materials and Design, University of Cambridge, United Kingdom \\ ${ }^{b}$ Centre for Natural Material Innovation, University of Cambridge, United Kingdom \\ *dus20@cam.ac.uk
}

\begin{abstract}
The use of laminated bamboo in structural applications is increasing, but the failure mechanisms of the material need further investigation. This study investigated the failure of laminated bamboo under compressive loading using digital image correlation (DIC). Notably, the unique speckled appearance of a bamboo transverse section, resulting from the spatial distribution of vascular fibre bundles in the parenchyma matrix, was directly used for DIC; no sample preparation was necessary. A $45^{\circ}$ degree shear face originated from the joint between several strips and led to the ultimate failure of the samples. However, when the strain exceeded $\sim 30 \%$ the analysis broke down due to sections of the specimen shearing off the sample. Our methodology demonstrates the potential of directly imaging laminated bamboo during mechanical testing to reveal complex full-field in-plane strain distribution, and the development and on-set of failure in the material.
\end{abstract}

Keywords: Biomaterials; Deformation and fracture; Laminated bamboo; Digital Image Correlation; Full-field strain visualization

\section{Introduction}

Bamboo is increasingly becoming a material used in construction due to global interest in lowering the environmental impact of construction. Bamboo forests can contain up to four times the carbon density per hectare than spruce forests due to higher carbon sequestration[1]. Alongside this, bamboo has mechanical properties 2-3 times greater than those of conventional timber[2 whilst being significantly lighter. In bamboo's natural form there is a large variation in cross-section and properties reducing the accuracy of modelling[3]. In order to form an applicable material, bamboo can be engineered into at least two forms: bamboo scrimber and laminated bamboo. Bamboo scrimber involves crushing fibre bundles, saturating them in resin then compressing it into a dense block, whilst maintaining longitudinal direction of fibres. In contrast, laminated bamboo retains more of the original culm matrix with strips of bamboo glued together to form sections [4].

The processing of bamboo culms to form laminated bamboo results in large beams of bamboo which can be cut into different sizes. Similarly, the size of the bamboo strips can be varied. Throughout this investigation the strips had average cross-section dimensions of $10 \mathrm{~mm} \mathrm{x}$ $6 \mathrm{~mm}$ and lengths often exceeding $1 \mathrm{~m}$, with the laminated bamboo having a moisture content of $\sim 8-10 \%$. Since laminated bamboo consists of individual strips, the impact of swelling and shrinkage is lower than that of solid wood panels [5]. However, in the initial stages of processing the bamboo culms are split into strips, usually around $2.5 \mathrm{~m}$ long, and the joining of these strips must be staggered to ensure there is not a concentrated region of weak (or stress-concentration) points. 
After destructive testing has been performed, digital image correlation (DIC) allows a greater depth of analysis to be carried out. In particular, it can be used to determine failure progression of samples. Work by Li et al. (2013) [6] used digital speckle correlation method to investigate the effect of age on bamboo specimen damage during testing and whether this technique could be used to predict the failure method of the sample. Similarly, Schoenmakers and Svensson (2011)[7] used non-contact measurements of timber samples to verify finite element models of single fastener connections. Previous work shows the interaction between loading forces to be complex but through DIC the failure surfaces can be investigated to a higher degree. This is an area that needs further advancement in bamboo research.

\section{Methodology}

Samples of approximate cross-section dimensions $21 \mathrm{~mm} \times 19 \mathrm{~mm}$, and width of $10 \mathrm{~mm}$ in strip direction were tested under compressive loading using an Instron 6025, retrofitted with a $5500 \mathrm{R}$ controller upgrade, with a $10 \mathrm{kN}$ loading head at a speed of $1 \mathrm{~mm} / \mathrm{min}$. This setup is seen in Figure 1, the sample is loaded in transverse compression with the strip (and bamboo grain) direction going into the page. During compression testing the sample was filmed enabling DIC to be performed on the samples using computer software [8]. The software works by identifying and tracking the movement of material within the sample allowing strains and displacements to be calculated, with the provision of length scales. Normally when using DIC a speckled pattern or random colouring of the sample would be used to provide the software with an image to analyse. In the case of bamboo under compression the vessel vascular fibre bundles are used as seeds, meaning that no modification or painting of the material is needed, which would have altered the surface properties of the material.

Images were taken at $5 \%$ strain intervals. The reference image was taken when the sample had not been loaded, i.e. a strain of $0 \%$, enabling the evolution of displacements during the testing to be found. The software works by taking subsections of the reference image and determining their location in the subsequent images, as well as any stretching or compression of the region. This will result in each image having a grid of displacements and strains which, when compiled, show the movement and strains of the sample during testing.

The computational cost can be modified by using a higher or lower resolution to the spacing between subsections. The higher the spacing the lower the computational cost, but the lower the accuracy. For this analysis the Green-Lagrangian strain tensor was used, over the Eulerian-Almansi tensor, as this is independent of rigid-body rotations and normal stretching from shear under low strains due to quadratic terms within the tensor matrix. However, at higher strains the analysis is likely to break-down mathematically as simple uniaxial strain is no longer exactly equal to $\frac{\Delta x}{x}$.

\section{Results and Discussion}

The relative values of epsilon, $\varepsilon_{x y}$, the local strain in $\mathrm{x}-\mathrm{y}$ direction, in a typical laminated bamboo material under transverse compression are presented in the full-field in-plane strain distribution images in Figure 3, at different absolute strain levels correlating to Figure 2. Due to the small size of our sample, and the relatively small width (into the page - Figure 1) of our sample, we ignore out-of-plane (x-z and $\mathrm{y}-\mathrm{z})$ deformation. For larger or thicker specimen, out-of-plane deformation may need to be accounted for (e.g. by the use of a second camera). During early stages of compression there is a $45^{\circ}$ shear face from the upper right corner 


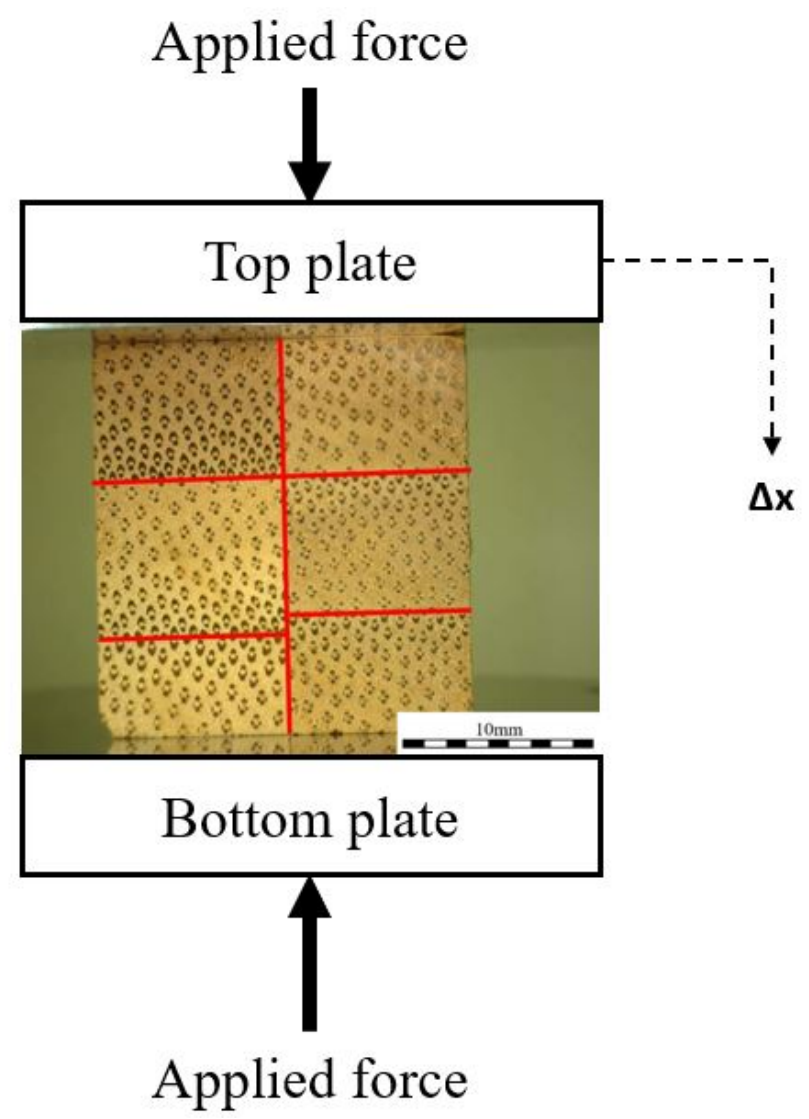

Figure 1: Typical sample with the joints between strips highlighted in red, with approximate dimensions $10 \mathrm{~mm} \times 21 \mathrm{~mm} \times 19 \mathrm{~mm}$, loaded in transverse compression.

leading towards the bottom left, as seen in Figure $3 a$ and $3 b$. The strain concentrations are focused around joints between strips. Note that as the strips are continuous along the length of the globally homogenous specimen, the stress-concentration (and thereafter failure) observed on the edge-surface of the transverse compression loaded sample is thought to be the same as in the middle of the sample. As the specimen is compressed further the shear face in the centre of the specimen connects with a high compressive strain area in the top right corner, Figure $3 \mathrm{c}$. This can be explained by a small chip from the sample detaching itself from the corner leading to a reduction in material in that region, therefore increasing the compressive strain experienced. As the specimen nears failure the strain is focused on two $45^{\circ}$ faces in the centre with the rest of the specimen experiencing strains close to 0 . One of these faces is in compression, dark blue lines in Figure $3 d$, whilst the other is in tension, dark red line. At this point, the absolute strain is 30\%, and from Figure 2 it is apparent that this is the onset of a second linear region, following a plateau between ca. $3 \%$ and $25 \%$ strain, where stress is relatively unchanged. This second linear region can be regarded as the onset of failure with the evident bipolar distribution in strain, i.e. some regions are highly strained, and the bulk of the material is not.

Above a strain of $30 \%$ DIC breaks down due to the shearing of parts of the specimen off the main body meaning that the software is unable to track the movement of material due to material failure. This can be seen in Figure 3 e. 


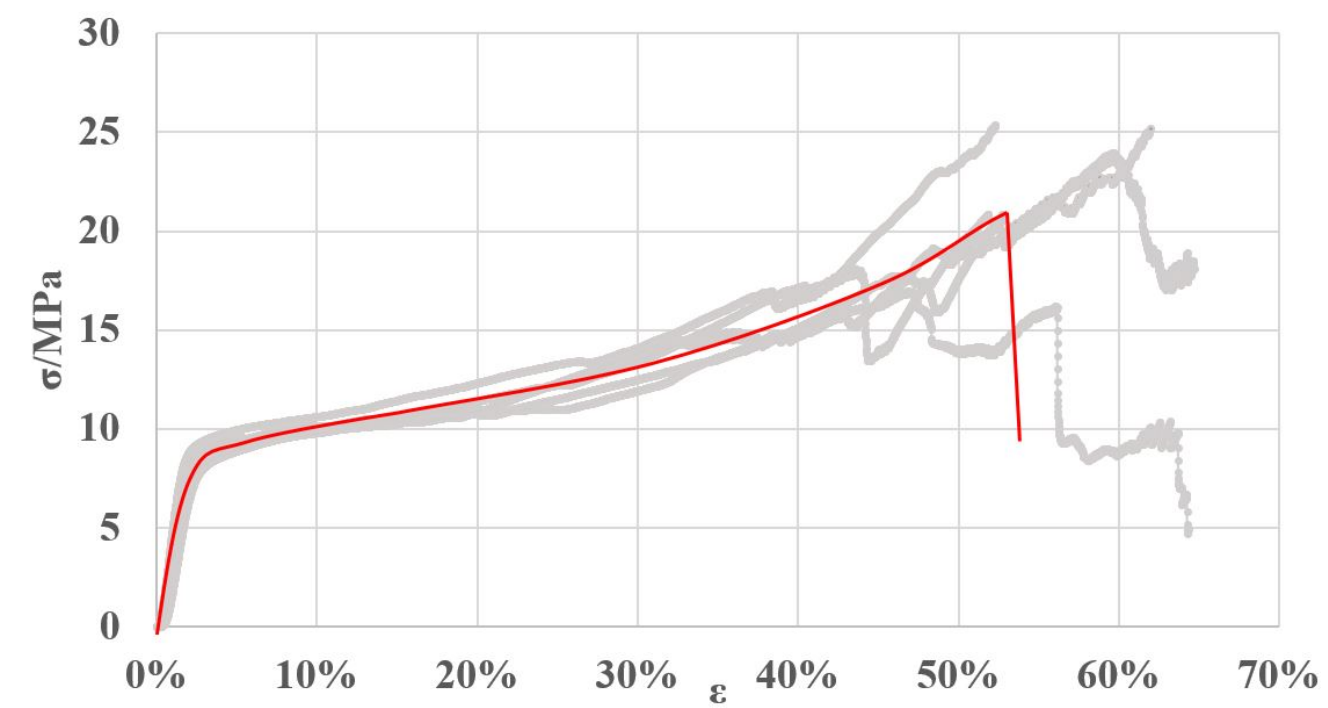

Figure 2: Absolute values of stress and strain found during testing, with the average characteristic curve shown in red.

\section{Conclusion}

Digital image correlation was performed on laminated bamboo during transverse compression testing. It enabled failure mechanisms of the samples to be identified and provided data up to strains of approximately 30\%, the onset of failure at the strip joints. Our findings reveal the opportunity the unique speckled surface of bamboo offers in experimental analysis of the material, and could be scaled to structural-scale samples using a combination of high-resolution images at the global scale and at specific regions of interest, with further improvements in observations possible by accounting for out-of-plane deformations.

\section{Acknowledgments}

The Dyson Bursary Fund part-funded the research for this project. Input from Simon Marshall and Graham Smith were invaluable during the testing process.

\section{References}

[1] Yiping, Lou, Yanxia, Li, Buckingham, Kathleen, Henley, G., Guomo, and Zhou. Bamboo and climate change mitigation, 012010.

[2] Stephane Schroder. What are the mechanical properties of bamboo?, November 2019.

[3] Perminder Kaur, K. Pant, Santosh Satya, and S. N. Naik. Bamboo: The material of the future. International Journal Series in Multidisciplinary Research, 2:17-24, 2016.

[4] Bhavna Sharma, Ana Gatoo, Maximilian Bock, and Michael Ramage. Engineered bamboo for structural applications. Construction and Building Materials, 81:66-73, 2015.

[5] Mahdavi, M., Clouston, P. L., Arwade, and S. R. Development of laminated bamboo lumber: Review of processing and performance and and economical considerations. Journal of Materials in Civil Engineering, 23(7):1036-1042, 2011.

[6] X Z Li, H Q Ren, Q Y Zhou, and Y Zhong. Application of digital speckle correlation method for examining deformation behaviour of moso bamboo (phyllostachys pubescens). Journal of Tropical Forest Science, 25(3):317-324, 2013. 
[7] J. (Dennis) Schoenmakers and S. Svensson. Embedment tests perpendicular to the grain - optical measurements of deformation fields. European Journal of Wood and Wood Products, 69(1):133-142, 2011.

[8] J. Blabar, B. Adair, and A. Antoniou. Open-source 2d digital image correlation matlab software. Experimental Mechanics, 55(6):1105-1122, 2015. 

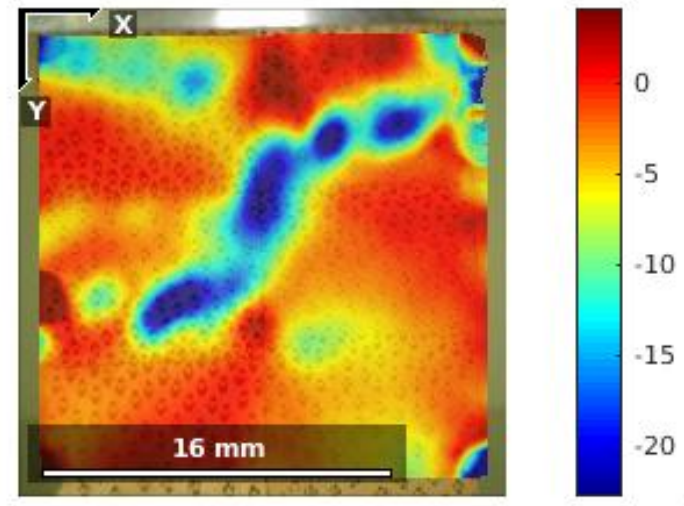

(a) Strain of $5 \%$.
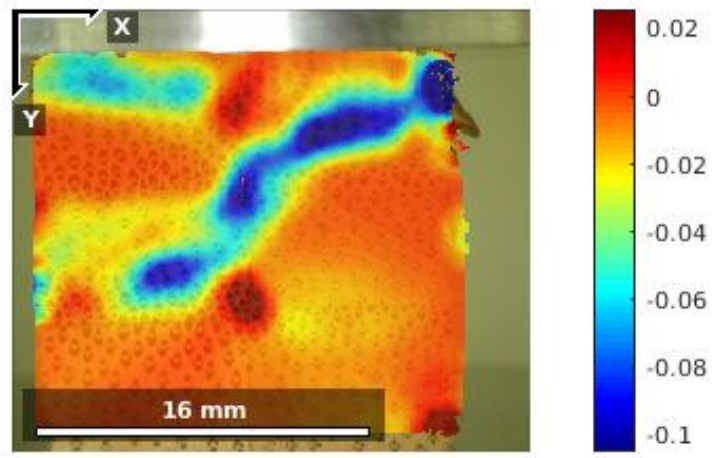

(c) Strain of $15 \%$.

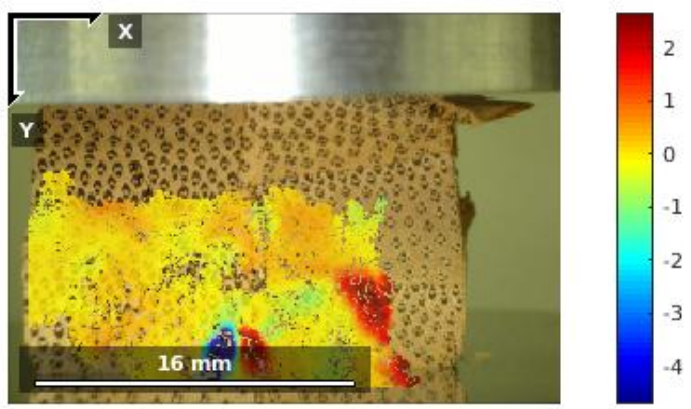

(e) Strain of $35 \%$.

Figure 3: $\varepsilon_{x y}$, local strain in the $x$-y direction, is observed in these in-plane full-field strain distribution images resulting from DIC analysis performed at medium strains, with the strain scale in absolute values.

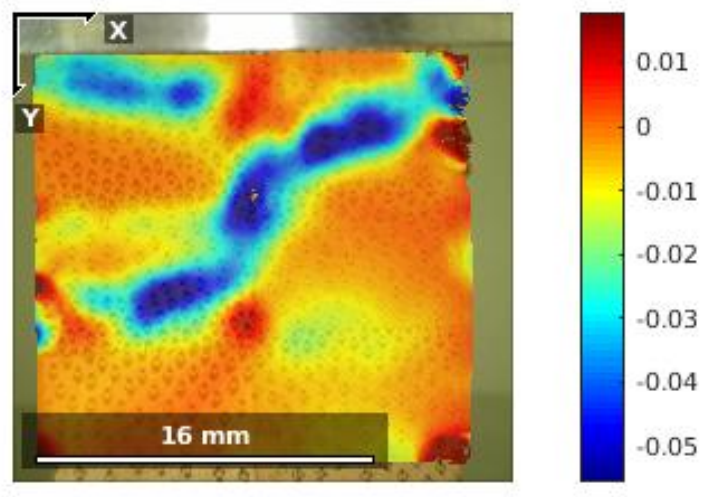

(b) Strain of $10 \%$.

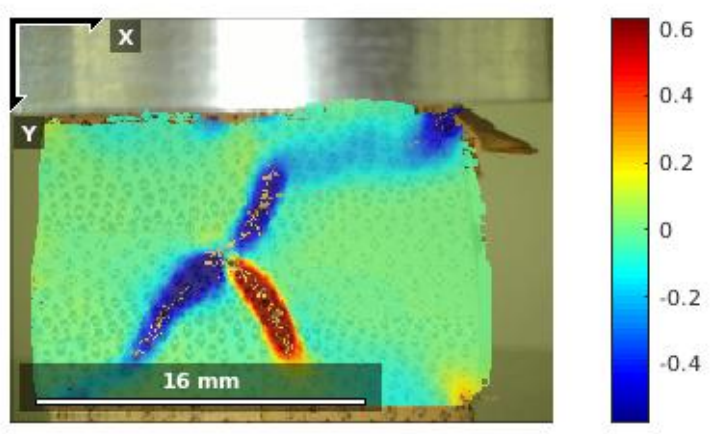

(d) Strain of $30 \%$. 